\title{
THE AND/OR THEOREM FOR PERCEPTRONS
}

\author{
I. D. BRUCE and D. EASDOWN
}

(Received 7 March 1990)

Communicated by T. E. Hall

\begin{abstract}
Minsky and Papert claim that, for any positive integer $n$, there exist predicates of order 1 whose conjunction and disjunction have order greater than $n$. Their proof is amended and a stronger result obtained of which their claim is a special case.
\end{abstract}

1991 Mathematics subject classification (Amer. Math. Soc.): 68 G 99.

\section{Introduction}

The perceptron is a simple parallel computing device, and its capabilities and limitations have been studied by Minsky and Papert [2]. They claim [2, Section 1.5] to have shown that for any positive integer $n$ there exist predicates $\psi_{1}$ and $\psi_{2}$ of order 1 for which both $\psi_{1} \wedge \psi_{2}$ and $\psi_{1} \vee \psi_{2}$ have order greater than $n$. This is called the AND/OR Theorem. Their argument [2, Chapter 4] establishes the existence of predicates $\psi_{1}$ and $\psi_{2}$ of order 1 for which $\psi_{1} \wedge \psi_{2}$ has order greater than $n$. A similar argument establishes the existence of predicates $\psi_{1}^{\prime}$ and $\psi_{2}^{\prime}$ of order 1 for which $\psi_{1}^{\prime} \vee \psi_{2}^{\prime}$ has order greater than $n$. However the arguments do not seem to guarantee that $\psi_{1}=\psi_{1}^{\prime}$ and $\psi_{2}=\psi_{2}^{\prime}$.

We amend their proof by adapting their techniques to prove a more general result, of which the AND/OR Theorem is a special case.

(C) 1992 Australian Mathematical Society 0263-6115/92 \$A2.00+0.00 


\section{Preliminaries}

Let $R$ be a finite set. A predicate on $R$ is a function $\phi$ of subsets of $R$ whose values, depending on the context, may be thought of as TRUE and FALSE, or 1 and 0 . To pass conveniently between these two kinds of predicate values, square bracket notation is used: if $\phi(X)$ is a statement about $X$ then

$$
[\phi(X)]= \begin{cases}1 \text { if } \phi(X) & \text { is true. } \\ 0 \text { if } \phi(X) & \text { is false. }\end{cases}
$$

Let $\Phi$ be a family of predicates on $R$, which is finite since $R$ is finite. A predicate $\psi$ is called a linear threshold function with respect to $\Phi$ if there are numbers $\theta$, and $\alpha_{\phi}$ for each $\phi \in \Phi$ such that

$$
\psi(X)=\left[\sum_{\phi \in \Phi} \alpha_{\phi} \phi(X)>\theta\right] .
$$

Let $L(\Phi)$ denote the set of linear threshold functions with respect to $\Phi$.

The support $S(\phi)$ of a predicate $\phi$ is the smallest subset $S$ of $R$ for which

$$
(\forall X \subseteq R) \phi(X)=\phi(X \cap S)
$$

The support exists because $(*)$ is satisfied when $S=R$ and if any (finite) collection of subsets $S$ satisfies $(*)$ then their intersection also satisfies $(*)$. Note however that if $R$ is allowed to be infinite then the notion of support need not make sense.)

The order of a predicate $\psi$ is the smallest number $k$ for which there is a set $\Phi$ of predicates for which $\psi \in L(\Phi)$ and $|S(\phi)| \leq k$ for all $\phi \in \Phi$.

If $A \subseteq R$ then the mask of $A$, denoted $\mu_{A}$, is the predicate defined by

$$
\mu_{A}(X)= \begin{cases}1 & \text { if } A \subseteq X \\ 0 & \text { otherwise. }\end{cases}
$$

It is easy to see that $S\left(\mu_{A}\right)=A$. If $A=\{a\}$ denote $\mu_{A}$ also by $\mu_{a}$.

THeOREM $1[2,1.5 .3]$. A predicate $\psi$ has order $k$ if and only if $k$ is the smallest number for which there exists a set $\Phi$ of masks such that $\psi \in L(\Phi)$ and $|S(\phi)| \leq k$ for all $\phi \in \Phi$.

Denote the (symmetric) group of all permutations of a set $X$ by $\operatorname{Sym}(X)$. Let $\Gamma$ be a subgroup of $\operatorname{Sym}(R)$. If $\psi$ is a predicate on $R$ and $\gamma \in \Gamma$, then define the predicate $\psi \gamma$ by

$$
\psi \gamma(X)=\psi(\gamma(X)) \text { for } X \subseteq R .
$$


Call predicates $\psi$ and $\phi \Gamma$-equivalent, written $\psi \equiv \phi$, if $\psi=\phi \gamma$ for some $\gamma \in \Gamma$. The Relation $\equiv$ is an equivalence because $\Gamma$ is a group. Note that, for masks, if $A, B \subseteq R$ then $\mu_{A} \gamma=\mu_{\gamma^{-1}(A)}$ for any $\gamma \in \Gamma$ so that

$$
\mu_{A} \equiv \mu_{B} \quad \text { if and only if } A=B \gamma \quad \text { for some } \gamma \in \Gamma \text {. }
$$

Call a predicate $\psi$ invariant under $\Gamma$ or $\Gamma$-invariant if

$$
\psi=\psi \gamma \text { for all } \gamma \in \Gamma \text {. }
$$

TheOREM 2 (Group Invariance Theorem) [2, 2.3]. Let $R$ be a finite set, $\Gamma$ a subgroup of $\operatorname{Sym}(R)$ and $\Phi$ a set predicates on $R$ closed under $\Gamma$ containing $\mu_{\phi}$ the mask of the empty set. Suppose that $\psi \in L(\Phi)$ and that $\psi$ is $\Gamma$-invariant. Then

$$
\psi=\left[\sum_{\phi \in \Phi} \beta_{\phi} \phi>0\right]
$$

where the coefficients $\beta_{\phi}$ depend only on the $\Gamma$-equivalence class of $\phi$, that is, if $\phi \equiv \phi^{\prime}$ then $\beta_{\phi}=\beta_{\phi^{\prime}}$.

Note (for when we apply this theorem later) that if $\Phi$ is the set of masks of support size $\leq k$ then $\Phi$ is closed under $\Gamma$ and contains $\mu_{\phi}$.

Let $\psi_{1}, \ldots, \psi_{m}$ be a sequence of predicates or formulae. Define a collection $\operatorname{AND} / \mathrm{OR}\left(\psi_{1}, \ldots, \psi_{m}\right)$ of predicates or formulae inductively:

$$
\operatorname{AND} / \mathrm{OR}\left(\psi_{1}\right)=\left\{\psi_{1}\right\},
$$

$\operatorname{AND} / \mathrm{OR}\left(\psi_{1}, \ldots, \psi_{i+1}\right)=\left\{\psi \vee \psi_{i+1}, \psi \wedge \psi_{i+1} \mid \psi \in \operatorname{AND} / \mathrm{OR}\left(\psi_{1}, \ldots, \psi_{i}\right)\right\}$

$$
\text { for } i=1, \ldots, m-1 \text {. }
$$

Thus AND/OR $\left(\psi_{1}, \ldots, \psi_{m}\right)$ contains $2^{m-1}$ predicates or formulae, obtained from $\psi_{1}$ by successively conjoining or disjoining $\psi_{i}$ 's. For example $\operatorname{AND} / \mathrm{OR}\left(\psi_{1}, \psi_{2}\right)=\left\{\psi_{1} \vee \psi_{2}, \psi_{1} \wedge \psi_{2}\right\}$, and

$\operatorname{AND} / \mathrm{OR}\left(\psi_{1}, \psi_{2}, \psi_{3}\right)=\left\{\psi \vee \psi_{2} \vee \psi_{3},\left(\psi_{1} \vee \psi_{2}\right) \wedge \psi_{3}, \psi_{1} \wedge \psi_{2} \wedge \psi_{3},\left(\psi_{1} \wedge \psi_{2}\right) \vee \psi_{3}\right\}$

The following is a trivial but important observation: if $\psi_{i}(X)=\left\{\begin{array}{l}1 \\ 0\end{array}\right.$ for all $i=1, \ldots, m$ then $\psi(X)=\left\{\begin{array}{l}1 \\ 0\end{array}\right.$ for all $\psi$ in $\operatorname{AND} / \operatorname{OR}\left(\psi_{1}, \ldots, \psi_{m}\right)$.

The last result we shall need is an adaptation of [2, Lemma 1].

Theorem 3 (Compactness). Let $Q_{1}\left(x_{1}, \ldots, x_{m}\right), Q_{2}\left(x_{1}, \ldots, x_{m}\right), \ldots$ be an infinite sequence of nonzero polynomials of $m$ variables of degree at most $N$, and let $n_{1}, n_{2}, \ldots$ be an infinite increasing sequence of positive 
integers. Suppose $\phi: \mathbb{Z}^{m} \rightarrow\{0,1\}$ is a function (predicate) such that for each $i=1,2, \ldots$ we have for all integers $x_{1}, \ldots, x_{m}$ between $-n_{i}$ and $n_{i}$

$$
\phi\left(x_{1}, \ldots, x_{m}\right)=\left[Q_{i}\left(x_{1}, \ldots, x_{m}\right)>0\right] .
$$

Then there exists a nonzero polynomial $Q\left(x_{1}, \ldots, x_{m}\right)$ of degree $\leq N$ such that for all integers $x_{1}, \ldots, x_{m}$

$$
\phi\left(x_{1}, \ldots, x_{m}\right)=\left\{\begin{array}{lll}
1 & \\
& \text { implies } Q\left(x_{1}, \ldots, x_{m}\right) & \left\{\begin{array}{l}
\geq 0 \\
0
\end{array}\right. \\
&
\end{array}\right.
$$

Proof. Let $\mathbf{x}$ be a vector of coordinates ranging over all products of powers of $x_{1}, \ldots, x_{m}$ in which for each product the sum of the exponents is at most $N$. Polynomials in $x_{1}, \ldots, x_{m}$ of degree $\leq N$ are then dot products $\mathbf{x} \cdot \mathbf{c}$ where $\mathbf{c}$ is a vector of constant coefficients. Thus for each $i$

$$
Q_{i}\left(x_{1}, \ldots, x_{m}\right)=\mathbf{x} \cdot \mathbf{c}_{i} \text { for some } \mathbf{c}_{i} .
$$

But the set $\left\{\hat{\mathbf{c}}_{i}=\mathbf{c}_{i} /\left\|\mathbf{c}_{i}\right\| \mid i=1,2, \ldots\right\}$ lies on the surface of the unit hypersphere which is compact [1], so has a limit point $c$ of length 1 . In particular c $\neq 0$. Let $Q\left(x_{1}, \ldots, x_{m}\right)=\mathbf{x} \cdot \mathbf{c}$, so $Q$ is nonzero of degree $\leq N$. Let $x_{1}, \ldots, x_{m}$ be any integers. Choose $n_{i}$ larger than each of $\left|x_{1}\right|, \ldots,\left|x_{m}\right|$. Suppose $\phi\left(x_{1}, \ldots, x_{m}\right)=1$, so by our hypothesis $Q_{j}\left(x_{1}, \ldots, x_{m}\right)>0$ for $j \geq i$. Thus $\mathbf{x} \cdot \mathbf{c}_{j}>0$, so $\mathbf{x} \cdot \hat{\mathbf{c}}_{j}>0$ for $j \geq i$. If $\mathbf{x} \cdot \mathbf{c}<0$ then choose $j \geq i$ such that

$$
\left\|\hat{\mathbf{c}}_{j}-\mathbf{c}\right\|<\frac{|\mathbf{c} \cdot \mathbf{c}|}{2\|\mathbf{x}\|}
$$

and so

$$
\begin{aligned}
\mathbf{x} \cdot \hat{\mathbf{c}}_{j} & =\mathbf{x} \cdot \mathbf{c}+\mathbf{x} \cdot\left(\hat{\mathbf{c}}_{j}-\mathbf{c}\right) \leq \mathbf{x} \cdot \mathbf{c}+\left|\mathbf{x} \cdot\left(\hat{\mathbf{c}}_{j}-\mathbf{c}\right)\right| \\
& \leq \mathbf{x} \cdot \mathbf{c}+\|\mathbf{x}\|\left\|\hat{\mathbf{c}}_{j}-\mathbf{c}\right\| \quad \text { (by the Cauchy-Schwarz inequality) } \\
& <\mathbf{x} \cdot \mathbf{c}+\frac{|\mathbf{x} \cdot \mathbf{c}|}{2}=\frac{\mathbf{x} \cdot \mathbf{c}}{2}<0,
\end{aligned}
$$

a contradiction. Hence $\mathbf{x} \cdot \mathbf{c} \geq 0$.

Similarly, if $\phi\left(x_{1}, \ldots, x_{m}\right)=0$ then $Q_{j}\left(x_{1}, \ldots, x_{m}\right) \leq 0$ for $j \geq i$, and in the same way one shows that $\mathbf{x} \cdot \mathbf{c} \leq 0$. This completes the proof of Theorem 3. 


\section{The main theorem}

The purpose of this section is to prove

THEOREM 4. Let $N$ and $m$ be any positive integers, $m \geq 2$. There exist predicates $\psi_{1}, \ldots, \psi_{m}$ of order 1 such that every predicate in

$$
\operatorname{AND} / \mathrm{OR}\left(\psi_{1}, \ldots, \psi_{m}\right)
$$

has order greater than $N$.

Then Minsky and Papert's claim follows.

CoRollary 5 (the AND/OR theorem). Let $N$ be any positive integer. There exist predicates $\psi_{1}$ and $\psi_{2}$ of order 1 such that both $\psi_{1} \wedge \psi_{2}$ and $\psi_{1} \vee \psi_{2}$ have order greater than $N$.

Proof of Theorem 4. To prove Theorem 4, let $n$ be a positive integer and $R$ any set containing $2 \mathrm{~nm}$ elements. Express $R$ as the disjoint union

$$
R=A_{1} \cup \cdots \cup A_{m} \text { where }\left|A_{i}\right|=2 n \text { for each } i .
$$

Define the predicate $\psi_{i}$, for $i=1$ to $m$, by

$$
\psi_{i}(X)=\left[\left|X \cap A_{i}\right| \geq n\right] \text { for } X \subseteq R .
$$

Note that each $\psi_{i}$ has order 1 because

$$
\psi_{i}(X)=\left[\sum_{a \in A_{i}} \mu_{a}(X) \geq n\right] .
$$

Thus for each $n$ we have defined a sequence $\psi_{1}, \ldots, \psi_{m}$ of order 1 predicates. We will show that for some $n$ each member of $\operatorname{AND} / \mathrm{OR}\left(\psi_{1}, \ldots, \psi_{m}\right)$ has order larger than $N$.

Suppose to the contrary that for each $n$ there is a predicate in

$$
\operatorname{AND} / \mathrm{OR}\left(\psi_{1}, \ldots, \psi_{m}\right)
$$

of order $\leq N$. There are infinitely many $n$ and only $2^{m-1}$ formulae in $\operatorname{AND} / \operatorname{OR}\left(\psi_{1}, \ldots, \psi_{m}\right)$, so there exists at least one formula $\psi$ in

$$
\operatorname{AND} / \mathrm{OR}\left(\psi_{1}, \ldots, \psi_{m}\right)
$$

such that for some infinite sequence of positive integers $n_{1}<n_{2}<\cdots$ the predicates $\psi$ for $n=n_{1}, n_{2}, \ldots$ have order $\leq N$. 
For the time being fix $n=n_{j}$. Let $\Phi$ be the set of masks of support size $\leq N$. Then $\psi \in L(\Phi)$ by Theorem 1. Consider the group

$$
\Gamma=\left\{\gamma \in \operatorname{Sym}(R) \mid \gamma\left(A_{i}\right)=A_{i} \text { for } i=1, \ldots, m\right\} .
$$

Then each predicate $\psi_{i}$ is $\Gamma$-invariant since

$$
\left|X \cap A_{i}\right|=\left|\gamma\left(X \cap A_{i}\right)\right|=\left|\gamma(X) \cap \gamma\left(A_{i}\right)\right|=\left|\gamma(X) \cap A_{i}\right|
$$

for each $\gamma \in \Gamma$ and $X \subseteq R$. By a simple induction all members of

$$
\operatorname{AND} / \mathrm{OR}\left(\psi_{1}, \ldots, \psi_{m}\right)
$$

are $\Gamma$-invariant. Hence $\psi$ is $\Gamma$-invariant, so by Theorem 2,

$$
\psi=\left[\sum_{\phi \in \Phi} \beta_{\phi} \phi>0\right]
$$

for some coefficients $\beta_{\phi}$ which depend only on the $\Gamma$-equivalence class of $\phi$. Let

$$
\begin{aligned}
V=\left\{\mathbf{v}=\left(v_{1}, \ldots, v_{m}\right) \in \mathbb{Z}^{m} \mid 0 \leq v_{i}\right. & \leq 2 n \\
\text { for } i & \left.=1 \text { to } m \text { and } v_{1}+\cdots+v_{m} \leq N\right\} .
\end{aligned}
$$

Then

$$
\Phi=\bigcup_{\mathbf{v} \in V} \Phi_{\mathbf{v}}
$$

where $\Phi_{\mathrm{v}}=\left\{\right.$ masks $\mu_{A}\left|A \cap A_{i}\right|=v_{i}$ for $i=1$ to $\left.m\right\}$. Since $\left.\Gamma\right|_{A_{i}}=\operatorname{Sym}\left(A_{i}\right)$, the $\Phi_{v}$ are the equivalence classes of $\Phi$ under $\Gamma$ as $\mathbf{v}$ ranges over $V$. Hence

$$
\psi(X)=\left[\sum_{\phi \in \Phi} \beta_{\phi} \phi(X)>0\right]=\left[\sum_{v \in V} \beta_{v} N_{v}(X)>0\right]
$$

where $\beta_{v}=\beta_{\phi}$ for any $\phi \in \Phi_{v}$ and $N_{v}(X)$ is the number of masks in $\phi_{v}$ whose support is contained in $X$. But then

$$
N_{\mathrm{v}}(X)=\left(\begin{array}{c}
\left|X \cap A_{1}\right| \\
v_{1}
\end{array}\right) \cdots\left(\begin{array}{c}
\left|X \cap A_{m}\right| \\
v_{m}
\end{array}\right)
$$

where $\left(\begin{array}{c}m \\ r\end{array}\right)$ is by definition $m(m-1) \cdots(m-r+1) / r$ ! so that $\left(\begin{array}{c}m \\ r\end{array}\right)=0$ if $m<r$.

Hence each $N_{v}(X)$ is a polynomial in the $m$ variables $y_{1}=\left|X \cap A_{1}\right|, \ldots$, $y_{m}=\left|X \cap A_{m}\right|$ of degree $v_{1}+\cdots+v_{m} \leq N$. But a linear combination of polynomials of degree $\leq N$ is also a polynomial of degree $\leq N$, so

$$
\psi(X)=\left[P\left(y_{1}, \ldots, y_{m}\right)>0\right]
$$


for some polynomial $P\left(y_{1}, \ldots, y_{m}\right)$ of degree $\leq N$. Note that $0 \leq y_{i} \leq 2 n$ since $\left|A_{i}\right|=2 n$ for each $i$. Put $x_{i}=y_{i}-n$, so that $-n \leq x_{i} \leq n$ for each $i$. Now put

$$
Q_{j}\left(x_{1}, \ldots, x_{m}\right)=P\left(x_{1}+n, \ldots, x_{m}+n\right)
$$

which is a polynomial in $x_{1}, \ldots, x_{m}$ of degree $\leq N$. The subscript $j$ is to remind us that $n=n_{j}$. Thus

$$
\psi(X)=\left[Q_{j}\left(x_{1}, \ldots, x_{m}\right)>0\right]
$$

where $-n_{j} \leq x_{1}, \ldots, x_{m} \leq n_{j}$.

Define functions $\phi_{i}: \mathbb{Z}^{m} \rightarrow\{0,1\}$ for $i=1$ to $m$ by

$$
\phi_{i}\left(z_{1}, \ldots, z_{m}\right)= \begin{cases}1 & \text { if } z_{i} \geq 0 \\ 0 & \text { if } z_{i}<0\end{cases}
$$

Then $\psi(X)=\phi_{i}\left(x_{1}, \ldots, x_{m}\right)$ where as before $x_{i}=\left|X \cap A_{i}\right|-n$ for $i=1$ to $m$. Let $\phi \in \operatorname{AND} / \mathrm{OR}\left(\phi_{1}, \ldots, \phi_{m}\right)$ be built using $\wedge$ and $\vee$ in exactly the same way as $\psi$. Then $\phi: \mathbb{Z}^{m} \rightarrow\{0,1\}$ and for the $x_{i}$ defined above $\phi\left(x_{1}, \ldots, x_{m}\right)=\psi(X)$. Thus

$$
\phi\left(x_{1}, \ldots, x_{m}\right)=\left[Q_{j}\left(x_{1}, \ldots, x_{m}\right)>0\right]
$$

where $-n_{j} \leq x_{1}, \ldots, x_{m} \leq n_{j}$.

This holds for each $j$, so by Theorem 3 there exists a nonzero polynomial $Q\left(x_{1}, \ldots, x_{m}\right)$ of degree $\leq N$ such that

$$
\phi\left(x_{1}, \ldots, x_{m}\right)=\left\{\begin{array} { l } 
{ 1 } \\
{ 0 }
\end{array} \quad \text { implies } Q ( x _ { 1 } , \ldots , x _ { m } ) \quad \left\{\begin{array}{l}
\geq 0 \\
\leq 0
\end{array}\right.\right.
$$

for all integers $x_{1}, \ldots, x_{m}$.

Put $Q\left(x_{1}, \ldots, x_{m}\right)=x_{m}^{d} q\left(x_{1}, \ldots, x_{m-1}\right)+r\left(x_{1}, \ldots, x_{m}\right)$ where $q\left(x_{1}, \ldots, x_{m-1}\right)$ is a nonzero polynomial in $x_{1}, \ldots, x_{m-1}$ and $r\left(x_{1}, \ldots, x_{m}\right)$ is either the zero polynomial or a nonzero polynomial such that the highest power of $x_{m}$ appearing is less than $d$. Note that $\phi=\phi^{\prime} \wedge \phi_{m}$ or $\phi=\phi^{\prime} \vee \phi_{m}$ for some $\phi^{\prime} \in \operatorname{AND} / \operatorname{OR}\left(\phi_{1}, \ldots, \phi_{m-1}\right)$, and that for all $x_{m}$

$$
\phi^{\prime}\left(x_{1}, \ldots, x_{m}\right)= \begin{cases}1 & \text { if } x_{1}, \ldots, x_{m-1} \geq 0 \\ 0 & \text { if } x_{1}, \ldots, x_{m-1}<0 .\end{cases}
$$

If $\phi=\phi^{\prime} \wedge \phi_{m}$ choose $x_{1}, \ldots, x_{m-1} \geq 0$, whilst if $\phi=\phi^{\prime} \vee \phi_{m}$ choose $x_{1}, \ldots, x_{m-1}<0$ for which $q\left(x_{1}, \ldots, x_{m-1}\right) \neq 0$. In both cases

$$
\phi\left(x_{1}, \ldots, x_{m}\right)= \begin{cases}1 & \text { if } x_{m} \geq 0 \\ 0 & \text { if } x_{m}<0\end{cases}
$$

so that

$$
Q\left(x_{1}, \ldots, x_{m}\right)= \begin{cases}\geq 0 & \text { if } x_{m} \geq 0 \\ \leq 0 & \text { if } x_{m}<0\end{cases}
$$


If $d$ is even then

$$
\begin{aligned}
0 & \leq \lim _{x_{m} \rightarrow \infty} Q\left(x_{1}, \ldots, x_{m}\right)=\lim _{x_{m} \rightarrow \infty} x_{m}^{d} q\left(x_{1}, \ldots, x_{m-1}\right. \\
& =\lim _{x_{m} \rightarrow-\infty} x_{m}^{d} q\left(x_{1}, \ldots, x_{m-1}\right)=\lim _{x_{m} \rightarrow-\infty} Q\left(x_{1}, \ldots, x_{m}\right) \leq 0
\end{aligned}
$$

so that $\lim _{x_{m} \rightarrow \infty} Q\left(x_{1}, \ldots, x_{m}\right)=0$, which contradicts that $q\left(x_{1}, \ldots, x_{m-1}\right)$ $\neq 0$.

Hence $d$ is odd. Now, if $\phi=\phi^{\prime} \wedge \phi_{m}$ choose $x_{1}, \ldots, x_{m-1}<0$, whilst if $\phi=\phi^{\prime} \vee \phi_{m}$ choose $x_{1}, \ldots, x_{m-1} \geq 0$ for which $q\left(x_{1}, \ldots, x_{m-1}\right) \neq 0$. In the first case $\phi\left(x_{1}, \ldots, x_{m}\right)=0$ so that $Q\left(x_{1}, \ldots, x_{m}\right) \leq 0$ for all $x_{m}$, whilst in the second case $\phi\left(x_{1}, \ldots, x_{m}\right)=1$ so that $Q\left(x_{1}, \ldots, x_{m}\right) \geq 0$ for all $x_{m}$. But, since $d$ is odd,

$$
\lim _{x_{m} \rightarrow \infty} Q\left(x_{1}, \ldots, x_{m}\right)=-\lim _{x_{m} \rightarrow-\infty} Q\left(x_{1}, \ldots, x_{m}\right)
$$

so that in both cases $\lim _{x_{m} \rightarrow \infty} Q\left(x_{1}, \ldots, x_{m}\right)=0$, which again contradicts that $q\left(x_{1}, \ldots, x_{m-1}\right) \neq 0$. This completes the proof of Theorem 4 .

\section{References}

[1] Jerrold E. Marsden, Elementary Classical Analysis (W. H. Freeman, San Francisco 1974).

[2] Marvin Lee Minsky and Seymour Papert, Perceptrons: an introduction to computational geometry (MIT Press, Cambridge, Massachusetts 1988).

Curtin University

University of Sydney

GPO Box U1987

NSW 2006

Perth WA 6001

Australia

Australia 